\title{
Transcriptomic analysis identifies genes and pathways related to myrmecophagy in the Malayan pangolin (Manis javanica)
}

\author{
Jing-E Ma ${ }^{1}$ ， Lin-Miao Li ${ }^{1}$ ， Hai-Ying Jiang ${ }^{1}$, Xiu-Juan Zhang ${ }^{1}$, Juan Li ${ }^{1}$ ， Guan-Yu Li ${ }^{1}$, Li-Hong Yuan ${ }^{1}$, Jun \\ Wu ${ }^{2}$, Jin-Ping Chen ${ }^{\text {Corresp. } 1}$ \\ ${ }^{1}$ Guangdong Key Laboratory of Animal Conservation and Resource, Guangdong Public Laboratory of Wild Animal Conservation and Utilization, Guangdong \\ Institute of Applied Biological Resources, Guangzhou, Guangdong, China \\ ${ }^{2}$ Wildlife disease surveillance and molecular ecology research Center, Nanjing Institute of Environmental Sciences under Ministry of Environmental \\ Protection, Nanjing, Jiangsu, China \\ Corresponding Author: Jin-Ping Chen \\ Email address: chenjp@gdei.gd.cn
}

The Malayan pangolin (Manis javanica) is an unusual, scale-covered, toothless mammal that specializes in myrmecophagy. Due to their threatened status and continuing decline in the wild, concerted efforts have been made to conserve and rescue this species in captivity in China. Maintaining this species in captivity is a significant challenge partly because little is known of the molecular mechanisms of its digestive system. Here, the first large-scale sequencing analyses of the salivary gland, liver and small intestine transcriptomes of an adult $M$. javanica genome were performed, and the results were compared with published liver transcriptome profiles for a pregnant $M$. javanica female. A total of 24,452 transcripts were obtained, among which 22,538 were annotated on the basis of seven databases. In addition, 3,373 new genes were predicted, of which 1,459 were annotated. Several pathways were found to be involved in myrmecophagy, including olfactory transduction, amino sugar and nucleotide sugar metabolism, lipid metabolism, and terpenoid and polyketide metabolism pathways. Many of the annotated transcripts were involved in digestive functions: 997 transcripts were related to sensory perception, 129 were related to digestive enzyme gene families, and 199 were related to molecular transporters. One transcript for an acidic mammalian chitinase was found in the annotated data, and this might be closely related to the unique digestive function of pangolins. These pathways and transcripts are involved in specialization processes related to myrmecophagy (a form of insectivory) and carbohydrate, protein and lipid digestive pathways, probably reflecting adaptations to myrmecophagy. Our study is the first to investigate the molecular mechanisms underlying myrmecophagy in $M$. javanica, and we hope that our results may play a role in the conservation of this species. 
1

2

3

4

5

6

7

8

9

10

11

12

13

14

15

16

17

18

19

20

21

22

23

24

25

26

27

28

29

\section{Transcriptomic analysis identifies genes and pathways related to myrmecophagy in the Malayan pangolin (Manis javanica)}

Jing-E Ma ${ }^{1}$, Lin-Miao Li ${ }^{1}$, Hai-Ying Jiang ${ }^{1}$, Xiu-Juan Zhang ${ }^{1}$, Juan Li ${ }^{1}$, Guan-Yu Li ${ }^{1}$, Li-Hong Yuan ${ }^{1}$, Jun $\mathrm{Wu}^{2}$, Jin-Ping Chen ${ }^{1^{*}}$

1Guangdong Key Laboratory of Animal Conservation and Resource Utilization, Guangdong Public Laboratory of Wild Animal

Conservation and Utilization, Guangdong Institute of Applied Biological Resources, Guangzhou, Guangdong, China

2 Nanjing Institute of Environmental Sciences under Ministry of Environmental Protection, Nanjing, Jiangsu, China

*Corresponding author

E-mail: chenjp@giabr.gd.cn

.

17

8

19


30 Abstract

The Malayan pangolin (Manis javanica) is an unusual, scale-covered, toothless mammal that specializes in myrmecophagy. Due to their threatened status and continuing decline in the wild, concerted efforts have been made to conserve and rescue this species in captivity in China.

Maintaining this species in captivity is a significant challenge partly because little is known of the molecular mechanisms of its digestive system. Here, the first large-scale sequencing analyses of the salivary gland, liver and small intestine transcriptomes of an adult $M$. javanica genome were performed, and the results were compared with published liver transcriptome profiles for a pregnant $M$. javanica female. A total of 24,452 transcripts were obtained, among which 22,538 were annotated on the basis of seven databases. In addition, 3,373 new genes were predicted, of which 1,459 were annotated. Several pathways were found to be involved in myrmecophagy, including olfactory transduction, amino sugar and nucleotide sugar metabolism, lipid metabolism, and terpenoid and polyketide metabolism pathways. Many of the annotated transcripts were involved in digestive functions: 997 transcripts were related to sensory perception, 129 were related to digestive enzyme gene families, and 199 were related to molecular transporters. One transcript for an acidic mammalian chitinase was found in the annotated data, and this might be closely related to the unique digestive function of pangolins. These pathways and transcripts are involved in specialization processes related to myrmecophagy (a form of insectivory) and carbohydrate, protein and lipid digestive pathways, probably reflecting adaptations to myrmecophagy. Our study is the first to investigate the molecular mechanisms underlying myrmecophagy in $M$. javanica, and we hope that our results may play a role in the conservation 
51 of this species.

\section{Introduction}

54 Pangolins, also known as scaly anteaters, are eutherians and placental mammals. Eight

pangolin species are recognized: four from Asia, Manis. javanica, M. pentadactyla, M.

crassicaudata, and M. culionensis, and four from Africa, Phataginus. tricuspis, P. tetradactyla,

Smutsia. gigantea, and S. temminckii, the genus Manis to the four Asian species while assigning

the two African tree pangolin species to the genus Phataginus and the two terrestrial African

pangolin species to the genus Smutsia (Du Toit et al. 2014; Gaudin et al. 2009). M. javanica is

found mainly in Southeast Asia (Trageser et al. 2017). In pangolins, unlike other placental

mammals, the skin is covered by large and overlapping keratinized scales (Meyer et al. 2013).

Furthermore, pangolins are edentulous or toothless and are thus specialized within an already

unusual mammalian dietary niche (Pietersen et al. 2015; Yang et al. 2007). Pangolins feed mainly

on ants and termites (Ashokkumar et al. 2017; Pietersen et al. 2015; Mahmood et al. 2013;

RICHER et al. 1997; Swart et al. 1999). Pangolins also have a well-developed muscular system

for fossorial or arboreal behavior and a remarkable olfactory system (Choo et al. 2016). As

predators of ants and termites, pangolins have a specialized diet and perform an important ecological role in regulating insect populations (Hua et al. 2015). Individual adult pangolins have been estimated to consume more than 70 million insects annually and pangolins provide an important regulatory function in controlling both ants and termites (Hua et al. 2015; Pietersen et 
72

73

economically important animals. Pangolins are the most poached and trafficked mammal in the world because of the high demand for their meat, which is considered a delicacy, and their scales are used in traditional medicine (Trageser et al. 2017).

Manis. javanica is classified as critically endangered according to the International Union for Conservation of Nature (IUCN) Red List of Threatened Species ${ }^{\circledR}$ (Challender et al. 2014) and has been included in appendix I of the Convention on International Trade in Endangered Species of Wild Fauna and Flora (CITES I). The numbers of $M$. javanica in the wild have been dramatically declining for several reasons. A major threat is the rapid loss and deterioration of their natural habitat as a result of deforestation; illegal hunting, which might reflect the pangolin's economic value; and human agricultural expansion (Hua et al. 2015). Therefore, artificial breeding may be the best choice for ensuring pangolin survival. A suitable artificial diet is one of the critical limiting factors in raising pangolins in captivity because they can have many digestive problems (Hua et al. 2015). Pangolins have adapted to a highly specialized diet of ants and termites; thus it is difficult to replace their natural food completely by artificial food (Hua et al. 2015; Yang et al. 2007). Pangolins favor high-protein, high-fat, and high-calorie food, and they have the notable ability to digest and absorb chitin in the digestive system (Hua et al. 2015; Yang et al. 2007). Chitin is a linear polymer of N-acetyl-beta-glucosaminidase subunits linked by a beta-1, 4 glycosidic bond and is one of the constituents of insect exoskeletons and the peritrophic membranes of ants (Kawashima et al. 2016). The internal degradation of chitin particles is mainly catalyzed by chitinase (Strobel et al. 2013).

Over the past 150 years, several zoos have tried to maintain pangolins. However, because of 
93

94

95

96

97

98

99

100

101

102

103

104

105

106

107

108

109

110

111

112

113

inadequate diets, these animals have not been successfully maintained for long periods by most zoos (Hua et al. 2015). Some formulas for diets fed to $M$. javanica in captivity use a paste mixture of several kinds of food, such as hard-boiled chicken eggs, multivitamin liquid, horse meat, water, mealworms, insectivore pellets, salmon oil, and powdered termite mound (Vijayan et al. 2009). Digestive disorders often appear in pangolins fed with artificial food, and the faeces of the animals become fluid. Several researchers have suggested that a certain proportion of chitin might be the key to artificial diets for pangolins (Ya-yong et al. 1999; Yang et al. 2007); however, an understanding of the molecular genetics that might provide a theoretical basis for raising $M$. javanica in captivity is lacking.

Genetic studies of endangered species have become increasingly widespread in the last two years (Choo et al. 2016; Mwale et al. 2017; Yusoff et al. 2016; Zhihai et al. 2016). In particular, the genomes and transcriptomes of M. javanica have been reported (Choo et al. 2016; Yusoff et al. 2016). Currently, increasing amounts of molecular information about M. javanica have become available as a result of the emergence of high-throughput next-generation sequencing (NGS) technologies. The complete genome sequencing of $M$. javanica and the transcriptome sequencing of eight organs, including the heart, liver, spleen, lung, kidney, thymus, cerebellum, and cerebrum, have progressively revealed unknown aspects of pangolin biology (Yusoff et al. 2016). Highquality transcriptomes have been used for analyses of the functional and phylogenetic aspects of immunity biology (Yusoff et al. 2016), but genetic research regarding myrmecophagy is still lacking. Here, two questions are raised. Are molecular pathways involved in the evolution of this dietary adaption for myrmecophagy, and how do they affect the appearance of this feature? In 
114 terms of dietary adaptation, we wanted to investigate whether specific genes exist for digestive 115 function. We selected the liver, small intestine, and salivary glands for transcriptome sequencing 116 to analyze the genetic selection of potential candidate genes involved in myrmecophagy. Salivary 117 glands are important amylase secretory organs although liver secretions also contain amylase and 118 lipase, and the small intestine is involved in the absorption of nutrients. We aimed to analyze the 119 unique feeding behavior of pangolins in the hope that our results may provide a new approach to 120 the protection of this species.

122 Materials and Methods

123 Ethics statement

All animal procedures were approved by the ethics committee for animal experiments at the Guangdong Institute of Applied Biological Resources (reference number: G2ABR20170523), and followed basic principles.

\section{Biological sample}

Briefly, an adult female $M$. javanica was provided by the Dongguan Institute of

Qingfengyuan Animal Medicine (Dongguan, Guangdong, China). The specimen was a wild individual that died in the process of rescue; the animal was $4.08 \mathrm{~kg}$ in weight before its natural death. The animal was dissected immediately after its natural death. During the dissection, ants were found in its stomach. The salivary glands, liver, and small intestine were collected as soon as possible, frozen in liquid nitrogen, and stored at $-80^{\circ} \mathrm{C}$ until RNA extraction. 
134

135

136

137

138

139

140

141

142

143

144

145

146

147

148

149

150

151

152

153

154

\section{RNA isolation, cDNA library construction and Illumina sequencing}

Total RNA was extracted from the three tissues using RNAiso reagent (Takara, Otsu, Japan)

and was treated with DNase I (Takara). RNA purity was checked using a NanoPhotometer

spectrophotometer (IMPLEN, CA, USA). RNA concentration was measured using the Qubit RNA

Assay Kit and a Qubit 2.0 Flurometer (Life Technologies, CA, USA), and RNA integrity was

assessed using the RNA Nano 6000 Assay Kit and the Agilent Bioanalyzer 2100 system (Agilent

Technologies, CA, USA). RNA was frozen at $-80^{\circ} \mathrm{C}$ until cDNA library construction.

RNA samples were then mixed with fragmentation buffer (Ambion) and fragmented into

short fragments; the average insert size was 200 bp. mRNA was purified from total RNA using

poly-T oligo-attached magnetic beads. DNA contaminants were further removed through DNase

enzyme digestion followed by rRNA removal. cDNA synthesis was then followed by PCR

amplification to generate a complete cDNA library, which was sent for sequencing in a flowcell

on the Illumina HiSeq ${ }^{\mathrm{TM}} 2000$ platform using the TruSeq PE Cluster Kit V3-cBot-HS (Illumina

PE-401-3001) and TruSeq SBS Kit-HS v3 200 cycles (Illumina FC-401-3001).

\section{Data assembly and annotation}

Four groups of sequencing data were used for assembly and annotation and the data were annotated using a published paper housed at the National Center for Biotechnology Information (NCBI) Sequence Read Archive (SRA) accession No.SRR2561213 (Yusoff et al. 2016). The primary sequencing data were cleaned by a) removing reads with adaptors; b) removing reads where the proportion of unknown bases $(N)$ exceeded $5 \%(-n=0.05)$; and c) removing low-quality reads (reads in which the proportion of bases with quality $<10$ exceeded $20 \%$, i.e., $-1=10$ and $-\mathrm{q}$ 
155 0.2). Clean reads were aligned to reference sequences using a spliced read mapper for RNA-Seq-

156 TopHat2 (http://ccb.jhu.edu/software/tophat/index.shtml), which is based on Bowtie2

157 (http://bowtie.cbcb.umd.edu/). The parameter values of RNA-Seq-TopHat2 were as follows; --

158 read-mismatches: 2, --read-edit-dist: 2, --max-intron-length: 5000000, --library-type: fr-

159 unstranded, --GTF: genome.gtf, --mate-inner-dist: 40, --solexa1.3-quals, and other parameters

160 were set to the default values. There were three steps: a) align the reads to the reference

161 transcriptomes; b) align the reads to the reference genome without the alignment described in a);

162 and c) align the reads segmentally to the reference genome without the alignment described in b).

163 Following this, the alignment data were used to calculate the distribution and coverage of the reads

164 on the reference genes.

165 To offer complementary insights, all transcripts $(>200 \mathrm{bp})$ were annotated on the basis of 166 basic local alignment search tool (BLASTX) results with e-values of $1 \mathrm{e}^{-5}$ against a total of seven 167 databases, including the non-redundant protein database (NR, ftp://ftp.ncbi.nih.gov/blast/db/); a 168 manually annotated, non-redundant protein sequence database (Swiss-Prot, 169 http://www.uniprot.org/); the Translated EMBL Nucleotide Sequence Data Library (TrEMBL, 170 http://www.bioinfo.pte.hu/more/TrEMBL.htm), which complements the Swiss-Prot protein 171 knowledgebase; the Kyoto Encyclopedia of Genes and Genomes (KEGG, 172 http://www.genome.jp/kegg/), which is used to understand the high-level functions and utilities of 173 biological system (such as the cell, the organism and the ecosystem) based on molecular-level 174 information, especially for large-scale molecular datasets generated by genome sequencing and 175 other high-throughput experimental technologies; Gene Ontology (GO, 
176

177

http://www.geneontology.org/), which described biological processes, molecular functions, and cellular components; and Clusters of Orthologous Groups of proteins (KOG/COG: COG, http://www.ncbi.nlm.nih.gov/COG/, KOG, http://www.ncbi.nlm.nih.gov/KOG/). The bestaligned results were used to determine the sequence direction of the transcripts. If the results from the different databases were in conflict, the sequence direction of the transcript was determined using the prioritization order NR, Swiss-Prot, KEGG and COG.

\section{Analysis of Differentially Expressed Genes}

Gene expression levels were estimated based on the fragments per kilo base $(\mathrm{kb})$ of transcript per million fragments mapped. The formula is as follows: FPKM=cDNA Fragments / Mapped Fragments (Millions) / Transcript Length (kb). Prior to differential gene expression analysis, for each sequenced library, the read counts were adjusted using the edgeR program package and a scaling normalized factor (Robinson et al. 2010). The differential expression analysis of two samples was performed using the DEGseq R package (Wang et al. 2010). P-value was adjusted

using q value, and q value $<0.005 \& \mid \log 2$ (fold change) $\mid \geqslant 1$ was set as the threshold for significantly differential expression (Storey \& Tibshirani 2003).

\section{Correlation between any two pangolin tissue transcriptomes}

To examine the similarity between the different organ transcriptomes, the expression levels of the transcripts (FPKM) in the transcriptomes of each tissue were manipulated using the tool "RSEM-calculate-expression" in the RSEM pipeline (http://deweylab.biostat.wisc.edu/RSEM), which performs accurate transcript quantification from RNA-Seq data with or without a reference genome. The reads for each tissue were mapped to the transcripts (Li \& Dewey 2011). Gene 
197 198 199 200

201

202

203

204

205

206

207

208

209

210

211

212

213

214

215

216

217

expression values, expressed as $\log 10(\mathrm{FPKM}+1)$ for the transcriptomic data from each tissue, were plotted against one another to produce scatter plots. $\mathrm{R}^{2}$ values were then calculated from the scatter plots to assess the correlation between any two $M$. javanica transcriptomes.

\section{Results}

\section{Illumina sequencing and assembly}

To obtain a comprehensive and representative transcriptome of $M$. javanica, 97,353,658 highquality clean reads (for a total length of 2,920,609,740 bp) were generated from the three tissues after the removal of the adaptor sequences. All high-quality sequencing reads from M. javanica are available on the NCBI Gene Expression Omnibus (GEO) database under accession numbers GSM2667949, GSM2667950 and GSM2667951. The average proportion of high-quality clean reads was $95.58 \%$ (Table 1). Clean reads were assembled into long assembled sequences (contigs) using TopHat2. The alignment efficiency between the sample and reference genome ranged from 69.57-89.30\% (Table 2), while the alignment efficiency between the sample and the exons of the reference genome ranged from 76.11-85.16\% (Figure S1).

\section{Functional annotation}

From the $M$. javanica transcriptome, 22,538 transcripts (93.05\%) were annotated on the basis of the COG, GO, KEGG, KOG, Swiss-Prot, TrEMBL, and NR databases using BLAST. A total of 6,228 transcripts were annotated against the COG database, followed by $13,977,14,115,16,648$, 17,135, and 20,964 transcripts annotated on the basis of the KEGG, KOG, GO, Swiss-Prot, and TrEMBL databases, respectively (Table S1). As expected, the majority of the 22,473 transcripts 
218 matched the NR databases (e-value $<10^{-5}$ ) (Figure S2 and File S1). The M. javanica transcripts 219 were annotated on the basis of the top BLASTX hits in the species distribution statistics. The top 220 five organisms were Ceratotherium simum (2,382 transcripts, 10.60\%), Equus caballus $(1,402$, 221 6.24\%), Canis lupus (1,349, 6.01\%), Mustela putorius (1,044, 4.65\%) and Odobenus rosmarus $222(1,022,4.55 \%)$ (Figure S3).

223 COG and KOG analysis

In the COG database, the largest category of $M$. javanica annotated transcripts was general 225 function prediction only (R) (2,438 transcripts, $27.76 \%)$, followed by replication, recombination, and repair $(\mathrm{L})(848,9.65 \%)$; transcription $(\mathrm{K})(845,9.62 \%)$; signal transduction mechanisms $(\mathrm{T})$ 227 (790, 8.99\%); and post-translational modification, protein turnover, and chaperones (O) (484, $5.51 \%$ ) (Figure S4A). In the KOG database, the largest category of $M$. javanica annotated transcripts was general function prediction only (R) (2,910 transcripts, $18.3 \%$ ), followed by signal transduction mechanisms $(\mathrm{T})(2,744,17.26 \%)$; post-translational modification, protein turnover, and chaperones $(\mathrm{O})(1,259,7.92 \%)$; function unknown $(\mathrm{S})(1,182,7.43 \%)$; and transcription $(\mathrm{K})$ $(1070,6.73 \%)$ (Figure S4B).

In both the COG and KOG analyses, several transcripts were involved in the transport and metabolism of the three major nutrients: 136 transcripts were related to carbohydrate transport and metabolism, 122 were related to lipid transport and metabolism, and 124 were related to amino acid transport and metabolism (File S2).

\section{Gene Ontology (GO)}


239

240

241

242

243

244

245

246

247

248

249

250

251

252

253

254

255

256

257

258

259

into 61 small classes in three ontologies: biological processes, molecular functions, and cellular components. A total of $44.36 \%$ of the transcripts were assigned to biological processes, $16.26 \%$ to molecular functions, and $39.38 \%$ to cellular components.

In the biological process ontology, the most highly represented terms were cellular processes $(10,494,63.03 \%)$, single-organism processes $(9,554,57.38 \%)$, and biological regulation $(7,986$, 47.97\%). The fourth most represented term was metabolic process $(7,173,43.08 \%)$, which was followed by response to stimulus $(4,915,29.52 \%)$, multicellular organismal process $(3,644$, $21.89 \%)$, signaling $(3,161,18.99 \%)$, localization $(3,001,18.03 \%)$, developmental process $(2,890$, $17.36 \%)$, and cellular component organization or biogenesis $(2,667,16.02 \%)$. The terms associated with biological regulation and metabolic process might be indicative of $M$. javanica transcriptome involvement in various digestive activities.

For molecular functions, the sequences were mainly assigned to binding $(9,371,56.29 \%)$ and catalytic activity $(5347,32.12 \%)$ followed by molecular transducer activity $(1,750,10.51 \%)$, receptor activity $(1,681,10.1 \%)$ and transporter activity $(1,026,6.16 \%)$, which might be involved in food digestion and absorption.

As anticipated, cell part $(11,445,68.74 \%)$ and cell $(11,412,68.54 \%)$ were the predominant terms assigned to the pangolin transcriptome in cellular components followed by organelle $(8,014$, $48.14 \%)$, membrane $(5,980,35.92 \%)$, membrane part $(4,441,26.67 \%)$, organelle part $(4,079$, $24.5 \%)$, macromolecular complex $(3,413,20.5 \%)$ and extracellular region $(1,044,6.27 \%)$ ( Figure S5, File S3). Overall, these results indicate that a broad range of biological activities were related to the expressed pangolin transcriptome, representing a pooled collection of the three digestive 
260

261

262

263

264

265

266

267

268

269

270

271

272

273

274

275

276

277

278

279

280

tissues sequenced.

\section{KEGG pathway analysis}

To identify the pathways in which the $M$. javanica transcripts were involved, the transcripts were mapped on the basis of KEGG pathways. A total of 13,977 (57.71\%) M. javanica transcripts were associated with 290 unique KEGG pathways, with a total of 15 unique KEGG pathways representing cellular processes, followed by 22, 27, 66, 68, and 89 representing genetic information passing, environmental information processing, organismal systems, human diseases, and metabolism, respectively (File S4).

The most-represented pathways in the $M$. javanica transcripts included olfactory transduction (969 transcripts) and pathways in cancer (444 transcripts), followed by the PI3K-Akt signaling pathway (391 transcripts), the MAPK signaling pathway (300 transcripts), and neuroactive ligandreceptor interaction (292 transcripts) (Figure S6). The sense of smell is closely related to the biological activity of instinctive behaviors such as feeding, and the olfactory pathway plays a key role in the specific recognition of smells, thus leading the animal to different foods. A total of 969 genes were associated with olfactory transduction in the M. javanica transcripts, 942 transcripts of which were annotated as various kinds of olfactory receptors. These findings may explain the keen sense of smell in M. javanica.

\section{Metabolic pathway analysis}

A total of 1,814 transcripts were associated with 89 unique KEGG metabolic pathways. Most transcripts were involved in lipid metabolism (431 transcripts), carbohydrate metabolism (365 transcripts), amino acid metabolism (321 transcripts), glycan biosynthesis and metabolism (271 
transcripts), nucleotide metabolism (233 transcripts), and the metabolism of cofactors and vitamins

282 (225 transcripts). Other transcripts were associated with global and overview maps (199

transcripts), energy metabolism (167 transcripts), the metabolism of other amino acids (119

transcripts), and xenobiotic biodegradation and metabolism (116 transcripts); a small number of

the transcripts were associated with terpenoid and polyketide metabolism (27 transcripts) and secondary metabolite biosynthesis (13 transcripts) (Fig. 1A).

287

\section{Carbohydrate metabolism}

Inositol phosphate metabolism (73 transcripts), glycolysis/gluconeogenesis (70 transcripts), starch and sucrose metabolism (57 transcripts), and amino sugar and nucleotide sugar metabolism (53 transcripts) were at the top of the carbohydrate metabolic lists, whereas ascorbate and aldarate metabolism were at the bottom.

The chitin-degrading enzyme acidic mammalian chitinase (CHIA), which is involved in the degradation of the chitin in the insect cuticle and the peritrophic membrane of the dietary ant, was found in the amino sugar and nucleotide sugar metabolism pathway (KEGG: 00520), thus suggesting that this pathway may be directly involved in ant digestion by M. javanica (Fig. 1B).

\section{Lipid metabolism}

Glycerophospholipid metabolism (100 transcripts), arachidonic acid metabolism (76 transcripts), steroid hormone biosynthesis (68 transcripts), and sphingolipid metabolism (55 transcripts) were at the top of the lipid metabolism list; in contrast, fatty acid biosynthesis (55 transcripts) was at the bottom. We identified transcripts from several pathways in unsaturated fatty acid metabolism, including arachidonic acid metabolism (76 transcripts), linoleic acid metabolism 
302 (36 transcripts), alpha-linolenic acid metabolism pathways (23 transcripts), and the biosynthesis

303 of unsaturated fatty acids (23 transcripts) (Fig. 1C).

304 Amino acid metabolism

305 Lysine degradation (66 transcripts), valine, leucine and isoleucine degradation (65

306

307

308

309

310

311

312

313

314

315

316

317

318

319

320

321

322

transcripts), arginine and proline metabolism (64 transcripts), and tryptophan metabolism (35

transcripts) were at the top of the amino acid metabolic lists. The biosynthesis pathways of some

amino acids, such as phenylalanine, tyrosine, and tryptophan (6 transcripts); valine, leucine and

isoleucine ( 5 transcripts); and lysine ( 2 transcripts) were at the bottom (Fig. 1D). None of the transcripts were found to be involved in arginine biosynthesis.

\section{Cofactors and vitamin metabolism and terpenoid and polyketide metabolism}

Retinol metabolism (65 transcripts), porphyrin and chlorophyll metabolism (46 transcripts), nicotinate and nicotinamide metabolism (36 transcripts), and pantothenate and CoA biosynthesis (31 transcripts) were at the top of the cofactors and vitamin metabolism lists. There was only one list relating to terpenoids and polyketides; terpenoid backbone biosynthesis (27 transcripts) (Fig. 1E, Fig. 2).

\section{Annotation of the new transcripts}

On the basis of the genome sequences of M. javanica, Cufflinks software was used to join the mapped reads, to compare them with the annotated information for the original genome, and to search for the gapped sequences, which were not annotated. A total of 3,373 new transcripts were discovered (File S5), of which 1,459 transcripts were annotated on the basis of the COG, GO, KEGG, KOG, Swiss-Prot, TrEMBL and NR databases using BLAST (Table S2 and File S6). 
In the Gene Ontology analysis, 75 new transcripts were involved in 22 metabolic categories.

324 Some of the new genes were involved in inositol metabolism (GO: 0006020; e.g.,

325 Manis_javanica_newGene_958), and some were involved in the linoleic acid metabolism (GO:

326 0043651; e.g., Manis_javanica_newGene_12722). For KEGG, 114 new genes were related to

327 metabolic function, including lipid metabolism (41 transcripts), carbohydrate metabolism (24

328 transcripts), cofactor and vitamin metabolism (22 transcripts), and amino acid metabolism (20

329 transcripts) (Figure S7).

Gene expression repertoire

Distributions of potential transcripts related to feeding among the three tissue libraries are

shown in Table 3, Table S3 and File S7, including the 997 transcripts related to sensory perception.

Of these transcripts, 972 were related to olfaction, and 11 and 14 transcripts were related to vision

and taste, respectively. A total of 133 transcripts were related to digestive enzyme gene families;

70 of these transcripts were related to lipid degradation, and 39 and 20 were related to the

degradation of proteins and carbohydrates, respectively. These genes were considered to be

involved in the profile of food choice, digestion and absorption, which might serve as a molecular

mechanism in myrmecophagy. Among these transcripts, acidic mammalian chitinase (CHIA),

chitinase-3-like protein 1 (CHI3L1), and chitinase domain-containing protein 1 (CHIDI) were

related to chitin degradation. A total of 199 transcripts were related to molecular transporters,

including sugar transporters, amino acid transporters, apolipoprotein transporters, cationic/anion

transporters, vitamin transporters, cotransporters and others; among these, the UDP-N- 
344 absorption of the chitin unit $\mathrm{N}$-acetylglucosamine.

345 Pairwise comparisons of different transcriptomic profiles

346 To examine the similarity among organ transcriptomes, we performed statistical correlation

347 analysis for each pair of organs using $\log 10(\mathrm{FPKM}+1)$ transformation to normalize the plots (Fig.

348 3). Our data showed that two liver transcriptome expression profiles were the most similar

$349\left(\mathrm{R}^{2}=0.53\right)$, followed by those of the liver and small intestine $\left(\mathrm{R}^{2}=0.30\right)$. The salivary glands and

small intestine had the least similar transcriptomic profiles $\left(\mathrm{R}^{2}=2 \mathrm{e}^{-0.4)}\right.$. The low correlation between

the liver transcriptomes found in our study and that of Yusoff et al. (2016) may reflect the varying

complexity between the same organs in different individuals, possibly because one of the two

specimens was pregnant. The differences between each pair of compared organs reflect the different digestive functions of the three organs.

A total of 11,055 transcripts were expressed (FPKM>1.0) in all three tissues; of these, 3,947

were annotated in KEGG. Several transcripts were enriched in lipid metabolism (214 transcripts),

carbohydrate metabolism (240 transcripts), and amino acid metabolism (188 transcripts) (Fig. 4).

Other transcripts near the top of the metabolic lists included glycerophospholipid metabolism (62

transcripts); valine, leucine and isoleucine degradation (53 transcripts); lysine degradation (49

transcripts); and inositol phosphate metabolism (45 transcripts). The biosynthesis pathways of

valine, leucine, and isoleucine (1 transcripts) and lysine (2 transcripts) were less commonly

represented.

Differentially expressed genes were identified among systems in the metabolic pathways of sugars, lipids, and amino acids. A total of 382 transcripts were differentially expressed between 
365 the small intestine and liver; this value compares with 258 transcripts that were differentially

366 expressed between the salivary glands and small intestine. Twenty-three and 27 genes differed

367 between the small intestine and liver for starch and sucrose metabolism and arginine and proline 368 metabolism, respectively, and 31 genes for steroid hormone biosynthesis differed between the liver 369 and the referred liver from Yusoff et al. 2016 (Figure S8).

370 Several transcripts were specifically expressed in a single sample, including 21 transcripts in the small intestine that were involved in 19 pathways, five transcripts in the liver that were involved in 11 pathways, 36 transcripts in the referred liver that were involved in 27 pathways, and six transcripts in the salivary glands that were involved in 10 pathways (Table S4). The highest number of specific transcripts was eight, and these transcripts were involved in the arachidonic acid metabolism in the liver reported in the previous study (Yusoff et al. 2016); six transcripts were involved in glycerophospholipid metabolism, in either lipid metabolism or arachidonic acid metabolism of the small intestine (Fig. 5).

\section{Discussion and conclusion} datasets, of which 1459 were annotated, and 75 new genes were involved in metabolism. The new genes provide new information for studying the myrmecophagous mechanisms of pangolins. A

large number of genes were expressed in all three tissues, and several specific genes in the three systems played different roles in the metabolism of sugars, lipids, and amino acids. The digestive 
386

387

388

389

390

391

392

393

394

395

396

397

398

399

400

401

402

403

404

405

406

between the small intestine and liver. The functions of the livers from the two different individuals differed in lipid metabolism pathways, suggesting that the ratio of lipid in the feed might be changed appropriately during pregnancy. Pangolin scales are considered an effective Chinese medicine and are thought to promote blood circulation, accelerate milk secretion, detumescence and apocenosis (Hua et al. 2015). The scales are helpful for the treatment of such human diseases.

Interestingly, our results indicated that several KEGG pathways were related to human diseases, possibly indicating the mechanisms by which pangolin scales act on human diseases. However, further data are needed to elucidate the underlying mechanisms.

Ants are a protein-rich food (Tomotake et al. 2010). They contain more than $50 \%$ crude protein, according to a nutritional value evaluation, and contain more than 20 amino acids; various microelements; special chemicals, such as formic acid and herbaceous acetaldehyde, which are triterpenoid compounds, and several vitamins (Pattarayingsakul et al. 2017). Many pangolin genes are likely to be involved in the digestion of these materials because 27 of the transcripts related to the terpenoid backbone biosynthesis, and this might be one of the biological basis for their adaptions to myrmecophagy. However, no genes were found to participate in the arginine synthesis pathway according to the KEGG analysis, and only two transcripts were involved in lysine synthesis. We did find a few transcripts encoding proteins that are necessary for the biosynthesis of amino acids that are essential in humans, such as lysine, valine, leucine, isoleucine, phenylalanine, and tryptophan (Galili et al. 2016; Zhenyukh et al. 2017). These results suggest that M. javanica might have at least a limited ability to synthesize those amino acids and that arginine might be the first limiting amino acid. Therefore, it might not be necessary to add the amino acids 
407 that pangolins have the potential to synthesis to manufactured feed.. However, the regulation of

408 the relevant transcripts is not fully characterized and the ability of pangolins to flourish without

409 those amino acids is unclear. This issue needs further research, which might be very useful for

410 theoretical and practical purposes.

411 In our M. javanica transcriptomic analysis, the same enriched GO terms were found to be

412 enriched in biological processes in response to a stimulus as those found by Yusoff et al. (2016),

413 indicating that the M. javanica transcripts may be actively involved in stimulus or stress responses.

414 However, several GO terms involving biological processes were found to be enriched in our study, including metabolic and developmental processes that might be involved in food digestion and absorption. Regarding molecular functions, receptor activity was similarly well enriched. In the previous study, the other main GO term found was cytoskeletal protein binding, and this likely required the evolution of a sophisticated musculoskeletal system or the formation of pangolin scales, which are made of keratin (Yusoff et al. 2016). In our study, some enriched GO terms were also related to metabolism, including catalytic activity, molecular transducer activity and transporter activity. The observed differences might be related to the different tissues used to obtain the transcriptome data. Chitin, one of the main components of the epidermis of ants and termites, comprises of Nacetyl-D-glucosamines (GluNAc) connected by a $\beta-1,4$ glycosidic bond. Chitin can be digested only with chitinase and acidic mammalian chitinase (AMCase); AMCase is widely found in the digestive organs of animals (Eurich et al. 2009; Krykbaev et al. 2010; Strobel et al. 2013). The origin of the digestive processes might be closely related to the activity of AMCase, which 
428 determines the beginning of chitin. The transporter genes of UDP-N-acetyl glucosamine 429 (SLC35A3, SLC35B4, and SLC35D2) might be directly related to the absorption of carbohydrate

430

434 units during chitin metabolism (Gerardy-Schahn et al. 2001). Therefore, chitin could be added to the formulated diets to aid in the digestion and absorption of nutrients. This suggestion is consistent with the referenced formulas mentioned in Vijayan et al. (2009).

Molecular adaptations consistent with the diet have also been shown in other studies. The tiger genome is particularly enriched in olfactory receptor activity, the G-protein-coupled receptor signaling pathway, signal transducer activity, amino-acid transport and protein metabolism. These markers of amino-acid metabolism have been associated with an obligatory carnivorous diet (Cho et al. 2013). We also found highly enriched transcripts for olfactory receptor activity in $M$. javanica. In red panda, regarding gene ontology and KEGG enriched analyses, significant terms and pathways were involved in limb development and nutrient utilization, including appendage and limb development, cilium assembly, protein digestion and absorption, and retinol metabolism; these pathways might be related to their bamboo diet (Hu et al. 2017). In the panda, loss-offunction is observed for $T A S 1 R 1$, and this might prevent the panda from expressing a functional umami taste receptor, thus partly explaining why the panda diet is primarily herbivorous despite its taxonomic classification as a carnivore ( $\mathrm{Li}$ et al. 2010). The polar bear is adapted to cope with a diet rich in fatty acids. Gene Ontology analysis for putative genes under positive selection in the polar bear lineage has shown that genes associated with adipose tissue development are enriched, reflecting the crucial role that lipids play in the ecology and life history of polar bears (Liu et al. 2014). However, transcripts involved in lipid metabolism were enriched in M. javanica, a finding 
449 that is generally consistent with the Gene Ontology analysis of the polar bear genome, while the 450 markers of amino-acid metabolism are apparently associated with the diet in pandas and tigers.

451 Malayan pangolins are endangered mammals for which captive breeding provides an 452 opportunity to study the molecular mechanisms of myrmecophagy. Our M. javanica transcriptomic 453 datasets of the three representative tissues present the first attempt at uncovering the mysteries of

454 455 456 457 458 459 460

transcriptome sequencing of digestive organs was performed to observe the metabolic pathways and functional genes related to myrmecophagy in an attempt to understand the molecular mechanisms involved. The results may provide an important theoretical basis for the successful captive breeding of this species. The transcriptomic data for the three organs were obtained with a high degree of confidence, and the transcripts were well annotated, thus providing a genomic and molecular basis for future study of this lesser-known mammalian species. However, the experimental design was constrained by the availability of samples, thus, further studies with more biological replicates are warranted. Other major organs involved in digestion (such as tongue, stomach, and pancreas) should be examined in further study, and these pangolin-specific genes may also be required to understand the unique traits or adaptations of pangolins (as compared to other mammals). Together, these results might shed light on the special diet of M. javanica.

\section{Acknowledgments}

We acknowledge BMKCloud (Beijing, China) for the data analysis. We acknowledge the Dongguan Institute of Qingfengyuan Animal Medicinal (Dongguan, Guangdong, China) for their 
470

471

\section{2}

473

474

475

476

477

478

479

480

481

482

483

484

485

486

487

488

489

490

491

492

493

494

495

496

497

498

499

500

501

502

503

504

505

506

507

508

selfless offering of three female pangolin samples.

\section{References}

Ashokkumar M, Valsarajan D, Suresh MA, and Kaimal ARaC, G. 2017. Stomach contents of the Indian Pangolin Manis crassicaudata (Mammalia: Pholidota: Manidae) in tropical forests of southern India. Journal of Threatened Taxa 9(5), pp:10246-10248.

Challender D, Nguyen Van T, Shepherd C, Krishnasamy K, Wang A, Lee, B., Panjang E, Fletcher, L., Heng S, Seah Han Ming J, Olsson A, Nguyen The Truong A, and Nguyen Van QC. 2014. Manis javanica. The IUCN Red List of Threatened Species 2014. Accessed on 15 August 2017. Version 2017-1. www.iucnredlist.org.

Cho YS, Hu L, Hou H, Lee H, Xu J, Kwon S, Oh S, Kim HM, Jho S, Kim S, Shin YA, Kim BC, Kim H, Kim CU, Luo SJ, Johnson WE, Koepfli KP, Schmidt-Kuntzel A, Turner JA, Marker L, Harper C, Miller SM, Jacobs W, Bertola LD, Kim TH, Lee S, Zhou Q, Jung HJ, Xu X, Gadhvi P, Xu P, Xiong Y, Luo Y, Pan S, Gou C, Chu X, Zhang J, Liu S, He J, Chen Y, Yang L, Yang Y, He J, Liu S, Wang J, Kim CH, Kwak H, Kim JS, Hwang S, Ko J, Kim CB, Kim S, Bayarlkhagva D, Paek WK, Kim SJ, O'Brien SJ, Wang J, and Bhak J. 2013. The tiger genome and comparative analysis with lion and snow leopard genomes. Nat Commun 4:2433. 10.1038/ncomms3433

Choo SW, Rayko M, Tan TK, Hari R, Komissarov A, Wee WY, Yurchenko AA, Kliver S, Tamazian G, Antunes A, Wilson RK, Warren WC, Koepfli KP, Minx P, Krasheninnikova K, Kotze A, Dalton DL, Vermaak E, Paterson IC, Dobrynin P, Sitam FT, Rovie-Ryan JJ, Johnson WE, Yusoff AM, Luo SJ, Karuppannan KV, Fang G, Zheng D, Gerstein MB, Lipovich L, O'Brien SJ, and Wong GJ. 2016. Pangolin genomes and the evolution of mammalian scales and immunity. Genome Res 26:1312-1322. 10.1101/gr.203521.115

Du Toit Z, Grobler, J.P., Kotzé, A., Jansen, R., Brettschneider, H. and Dalton, D.L. 2014. The complete mitochondrial genome of Temminck's ground pangolin (Smutsia temminckii; Smuts, 1832) and phylogenetic position of the Pholidota (Weber, 1904). Gene 551(1):49-54.

Eurich K, Segawa M, Toei-Shimizu S, and Mizoguchi E. 2009. Potential role of chitinase 3-like-1 in inflammationassociated carcinogenic changes of epithelial cells. World J Gastroenterol 15:5249-5259.

Galili G, Amir R, and Fernie AR. 2016. The Regulation of Essential Amino Acid Synthesis and Accumulation in Plants. Annu Rev Plant Biol 67:153-178. 10.1146/annurev-arplant-043015-112213

Gaudin TJ, Emry RJ, and Wible JR. 2009. The Phylogeny of Living and Extinct Pangolins (Mammalia, Pholidota) and Associated Taxa: A Morphology Based Analysis. Journal of Mammalian Evolution 16:235-305. 10.1007/s10914-009-9119-9

Gerardy-Schahn R, Oelmann S, and Bakker H. 2001. Nucleotide sugar transporters: Biological and functional aspects. Biochimie 83:775-782. Doi 10.1016/S0300-9084(01)01322-0

Hu Y, Wu Q, Ma S, Ma T, Shan L, Wang X, Nie Y, Ning Z, Yan L, Xiu Y, and Wei F. 2017. Comparative genomics reveals convergent evolution between the bamboo-eating giant and red pandas. Proc Natl Acad Sci U S A 114:1081-1086. 10.1073/pnas.1613870114

Hua L, Gong S, Wang F, Li W, Ge Y, Li X, and Hou F. 2015. Captive breeding of pangolins: current status, problems and future prospects. ZooKeys:99-114. 10.3897/zookeys.507.6970

Kawashima S, Ikehata H, Tada C, Ogino T, Kakizaki H, Ikeda M, Fukushima H, and Matsumiya M. 2016. Stomach Chitinase from Japanese Sardine Sardinops melanostictus: Purification, Characterization, and Molecular 
Cloning of Chitinase Isozymes with a Long Linker. Mar Drugs 14:22. 10.3390/md14010022

Krykbaev R, Fitz L, Reddy PS, Winkler A, Xuan DJ, Yang XK, Fleming M, and Wolf SF. 2010. Evolutionary and biochemical differences between human and monkey acidic mammalian chitinases. Gene 452:63-71. 10.1016/j.gene.2009.12.005

Li B, and Dewey CN. 2011. RSEM: accurate transcript quantification from RNA-Seq data with or without a reference genome. BMC Bioinformatics 12:323. Artn 323 10.1186/1471-2105-12-323

Li R, Fan W, Tian G, Zhu H, He L, Cai J, Huang Q, Cai Q, Li B, Bai Y, Zhang Z, Zhang Y, Wang W, Li J, Wei F, Li H, Jian M, Li J, Zhang Z, Nielsen R, Li D, Gu W, Yang Z, Xuan Z, Ryder OA, Leung FC, Zhou Y, Cao J, Sun X, Fu Y, Fang X, Guo X, Wang B, Hou R, Shen F, Mu B, Ni P, Lin R, Qian W, Wang G, Yu C, Nie W, Wang J, Wu Z, Liang H, Min J, Wu Q, Cheng S, Ruan J, Wang M, Shi Z, Wen M, Liu B, Ren X, Zheng H, Dong D, Cook K, Shan G, Zhang H, Kosiol C, Xie X, Lu Z, Zheng H, Li Y, Steiner CC, Lam TT, Lin S, Zhang Q, Li G, Tian J, Gong T, Liu H, Zhang D, Fang L, Ye C, Zhang J, Hu W, Xu A, Ren Y, Zhang G, Bruford MW, Li Q, Ma L, Guo Y, An N, Hu Y, Zheng Y, Shi Y, Li Z, Liu Q, Chen Y, Zhao J, Qu N, Zhao S, Tian F, Wang X, Wang H, Xu L, Liu X, Vinar T, Wang Y, Lam TW, Yiu SM, Liu S, Zhang H, Li D, Huang Y, Wang X, Yang G, Jiang Z, Wang J, Qin N, Li L, Li J, Bolund L, Kristiansen K, Wong GK, Olson M, Zhang X, Li S, Yang H, Wang J, and Wang J. 2010. The sequence and de novo assembly of the giant panda genome. Nature 463:311-317. 10.1038/nature08696

Liu S, Lorenzen ED, Fumagalli M, Li B, Harris K, Xiong Z, Zhou L, Korneliussen TS, Somel M, Babbitt C, Wray G, Li J, He W, Wang Z, Fu W, Xiang X, Morgan CC, Doherty A, O'Connell MJ, McInerney JO, Born EW, Dalen L, Dietz R, Orlando L, Sonne C, Zhang G, Nielsen R, Willerslev E, and Wang J. 2014. Population genomics reveal recent speciation and rapid evolutionary adaptation in polar bears. Cell 157:785-794. 10.1016/j.cell.2014.03.054

Mahmood T, Jabeen K, Hussain I, and Kayani AR. 2013. Plant Species Association, Burrow Characteristics and the Diet of the Indian Pangolin, Manis crassicaudata, in the Potohar Plateau, Pakistan. Pakistan Journal of Zoology 45:1533-1539.

Meyer W, Liumsiricharoen M, Suprasert A, Fleischer LG, and Hewicker-Trautwein M. 2013. Immunohistochemical demonstration of keratins in the epidermal layers of the Malayan pangolin (Manis javanica), with remarks on the evolution of the integumental scale armour. Eur J Histochem 57:e27. 10.4081/ejh.2013.e27

Mwale M, Dalton DL, Jansen R, De Bruyn M, Pietersen D, Mokgokong PS, and Kotze A. 2017. Forensic application of DNA barcoding for identification of illegally traded African pangolin scales. Genome 60:272-284. 10.1139/gen-2016-0144

Pattarayingsakul W, Nilavongse A, Reamtong O, Chittavanich P, Mungsantisuk I, Mathong Y, Prasitwuttisak W, and Panbangred W. 2017. Angiotensin-converting enzyme inhibitory and antioxidant peptides from digestion of larvae and pupae of Asian weaver ant, Oecophylla smaragdina, Fabricius. J Sci Food Agric 97:3133-3140. 10.1002/jsfa.8155

Pietersen DW, Symes CT, Woodborne S, McKechnie AE, and Jansen R. 2015. Diet and prey selectivity of the specialist myrmecophage, Temminck's ground pangolin. Journal of Zoology.

RICHER R, Coulson IAN, and HEATH M. 1997. Foraging behaviour and ecology of the Cape pangolin (Manis temminckii) in north - western Zimbabwe. African Journal of Ecology 35(4), pp:361-369.

Robinson MD, McCarthy DJ, and Smyth GK. 2010. edgeR: a Bioconductor package for differential expression analysis of digital gene expression data. Bioinformatics 26:139-140. 10.1093/bioinformatics/btp616 Storey JD, and Tibshirani R. 2003. Statistical significance for genomewide studies. Proc Natl Acad Sci U S A 
100:9440-9445. 10.1073/pnas.1530509100

Strobel S, Roswag A, Becker NI, Trenczek TE, and Encarnacao JA. 2013. Insectivorous bats digest chitin in the stomach using acidic mammalian chitinase. PLOS ONE 8:e72770. 10.1371/journal.pone.0072770

Swart JM, Richardson PRK, and Ferguson JWH. 1999. Ecological factors affecting the feeding behaviour of pangolins (Manis temminckii). Journal of Zoology 247:281-292.

Tomotake H, Katagiri M, and Yamato M. 2010. Silkworm pupae (Bombyx mori) are new sources of high quality protein and lipid. J Nutr Sci Vitaminol (Tokyo) 56:446-448.

Trageser SJ, Ghose A, Faisal M, Mro P, Mro P, and Rahman SC. 2017. Pangolin distribution and conservation status in Bangladesh. PLOS ONE 12:e0175450. 10.1371/journal.pone.0175450

Vijayan M, Yeong C, and Ling D. 2009. Captive management of Malayan pangolins Manis javanica in the Night Safari. WORKSHOP ON TRADE AND CONSERVATION OF PANGOLINS NATIVE TO SOUTH AND SOUTHEAST ASIA. p 119.

Wang L, Feng Z, Wang X, Wang X, and Zhang X. 2010. DEGseq: an R package for identifying differentially expressed genes from RNA-seq data. Bioinformatics 26:136-138. 10.1093/bioinformatics/btp612

Ya-yong K, Hong C, Shi-bao W, Qian L, and Gan-xin F. 1999. A study on Chinese pangolin's main food nutrition. Zoological Research 20:394-395.

Yang CW, Chen S, Chang CY, Lin MF, Block E, Lorentsen R, Chin JS, and Dierenfeld ES. 2007. History and dietary husbandry of pangolins in captivity. Zoo Biol 26:223-230. 10.1002/zoo.20134

Yusoff A, Tan TK, Hari R, Koepfli KP, Wee WY, Antunes A, Sitam FT, Rovie-Ryan JJ, Karuppannan KV, Wong GJ, Lipovich L, Warren WC, O'Brien SJ, and Choo SW. 2016. De novo sequencing, assembly and analysis of eight different transcriptomes from the Malayan pangolin. Sci Rep 6:28199. 10.1038/srep28199

Zhenyukh O, Civantos E, Ruiz-Ortega M, Sanchez MS, Vazquez C, Peiro C, Egido J, and Mas S. 2017. High concentration of branched-chain amino acids promotes oxidative stress, inflammation and migration of human peripheral blood mononuclear cells via mTORC1 activation. Free Radic Biol Med 104:165-177. 10.1016/j.freeradbiomed.2017.01.009

Zhihai H, Jiang X, Shuiming X, Baosheng L, Yuan G, Chaochao Z, Xiaohui Q, Wen X, and Shilin C. 2016. Comparative optical genome analysis of two pangolin species: Manis pentadactyla and Manis javanica. Gigascience 5:15. 10.1093/gigascience/giw001 


\section{Figure 1 (on next page)}

The metabolic pathway analysis of transcripts from Manis javanica.

The $\mathrm{x}$-axis shows the numbers of annotated transcripts in one class, and the $y$-axis shows the KEGG function classes. 


\section{(A)}

\section{Metabolism}

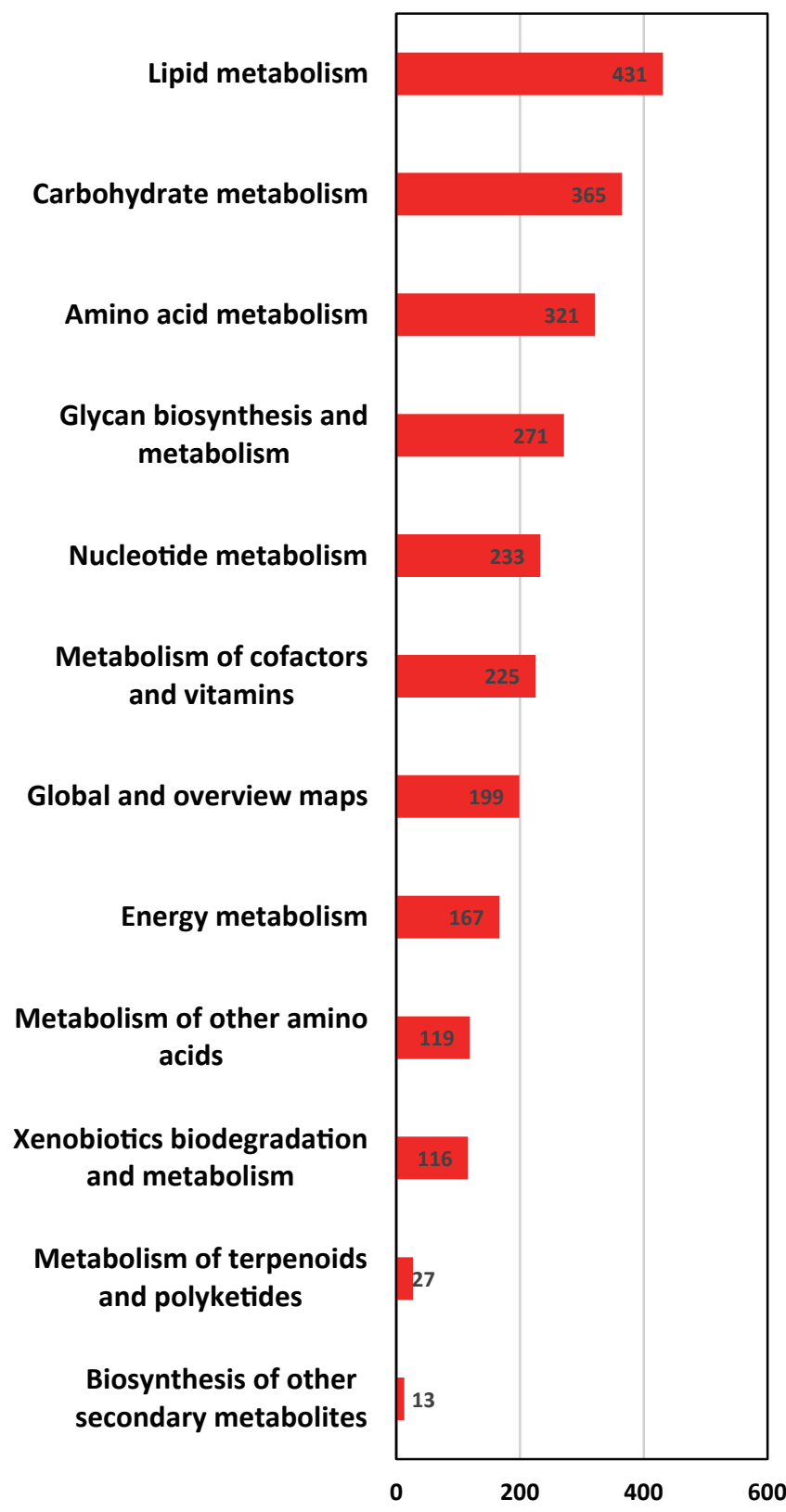

Annotated Transcripts number
(B) $\begin{array}{r}\text { Biosynthesis of unsaturated fatty adids } \\ \text { Linoleic acid metabolism }\end{array}$ Sphingolipid metabolism Glycerophospholipid metabolism Steroid hormone biosynthesis Steroid biosynthesis Fatty acid degradation Fatty acid biosynthesis
(C)

Glyoxylate and dicarboxylate metabolism Amino sugar and nucleotide sugar metabolism Ascorbate and aldarate metabolism Fructose and mannose metabolism Pentose phosphate pathway Glycolysis / Gluconeogenesis

(D)

Phenylalanine, tyrosine and tryptophan biosynthesis Phenylalanine metabolism Histidine metabolism Lysine degradation Valine, leucine and isoleucine biosynthesis Cysteine and methionine metabolism Biosynthesis of unsaturated fatty acids

(E)

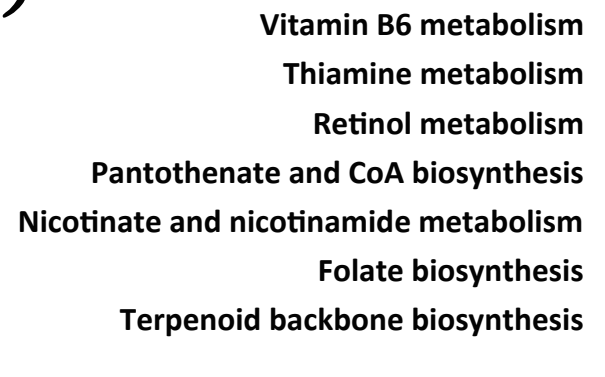

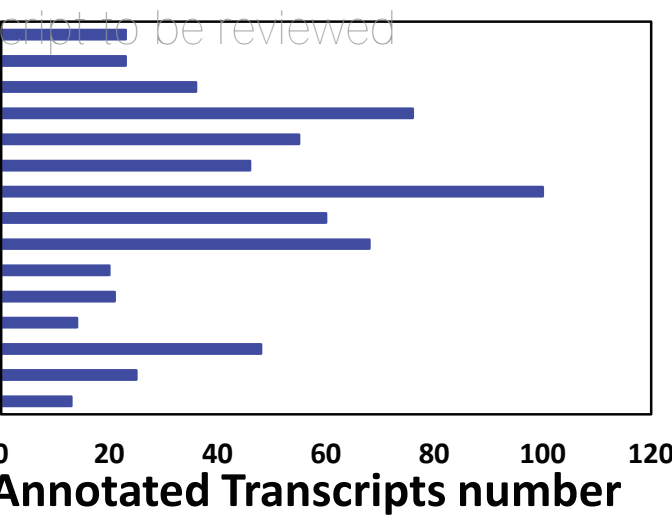

Lipid

metabolism

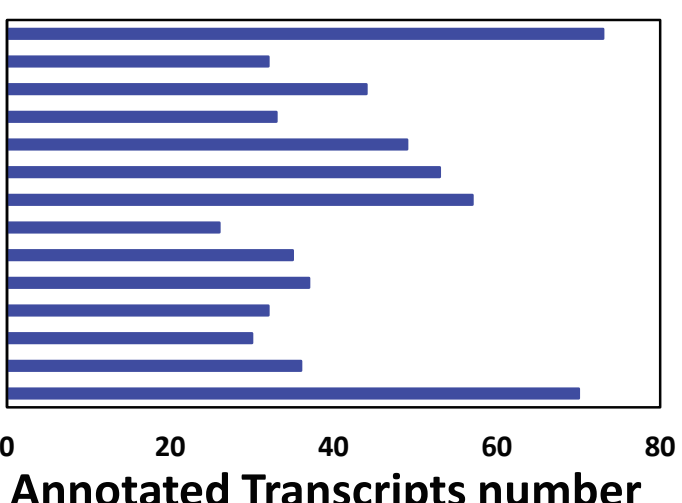

Carbohydrate metabolism
Amino acid metabolism

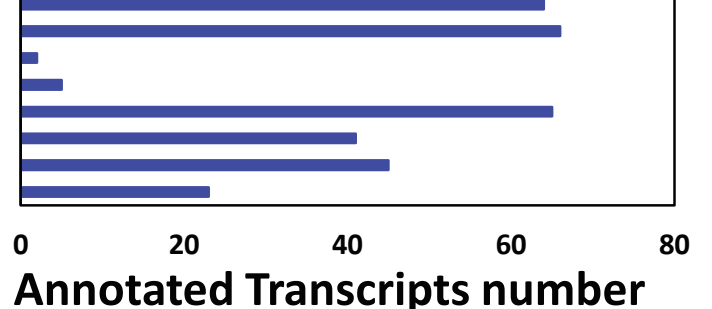

Annotated Transcripts number

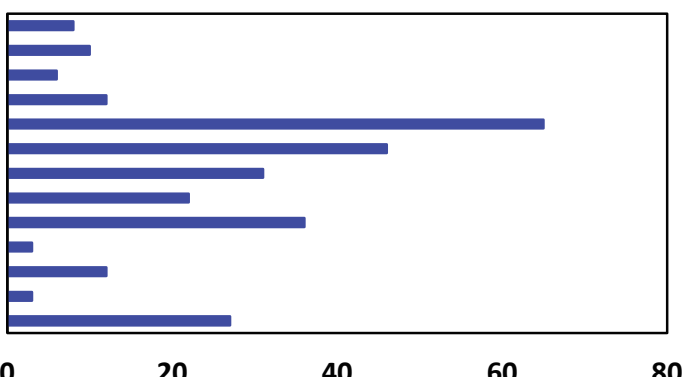

Metabolism of cofactors and vitamins, and terpenoids and polyketides 
Figure 2 (on next page)

Terpenoid backbone biosynthesis (KEGG map 00900). 


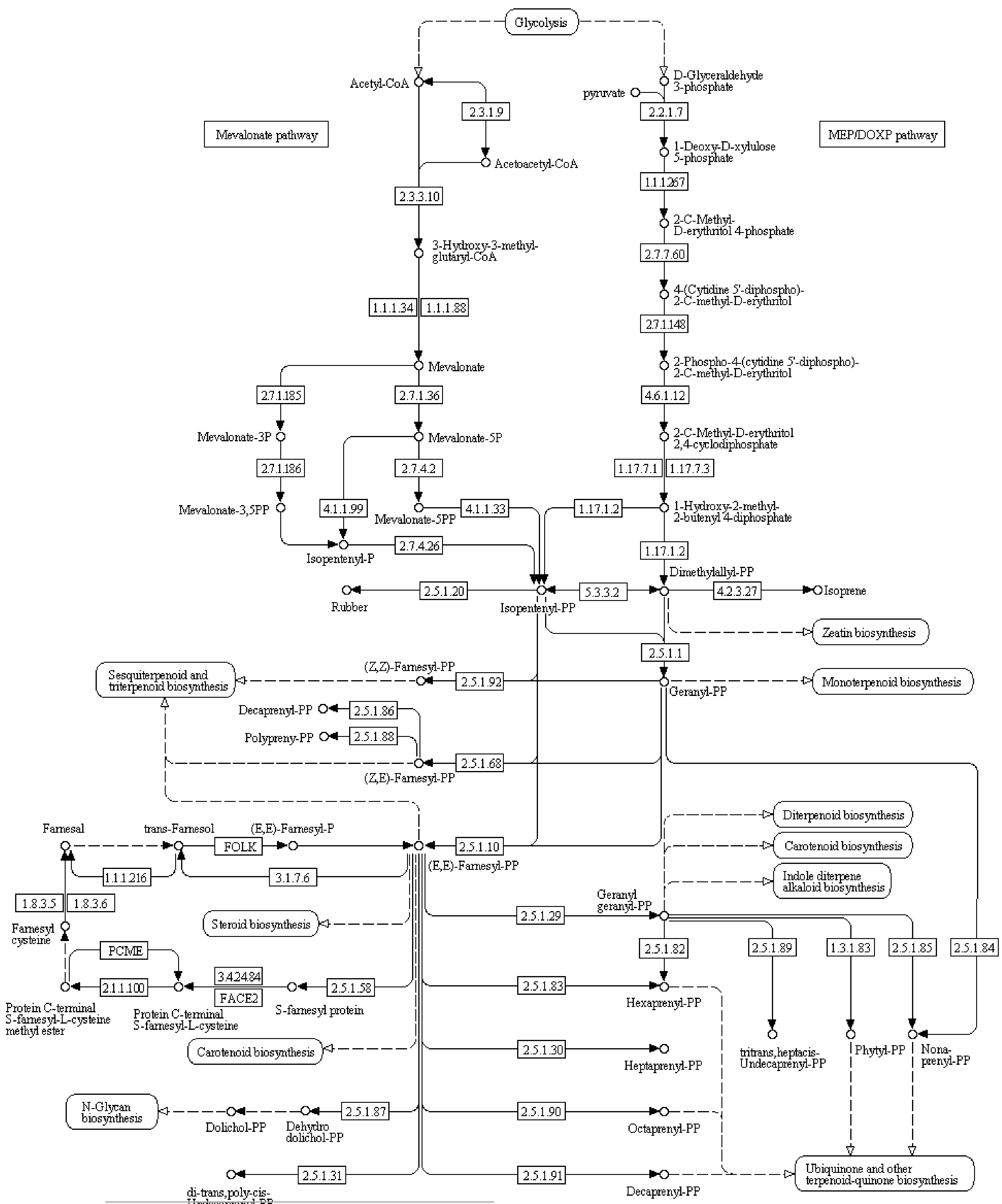




\section{Figure 3 (on next page)}

Pairwise correlation between organs. 
(A) PeerJ

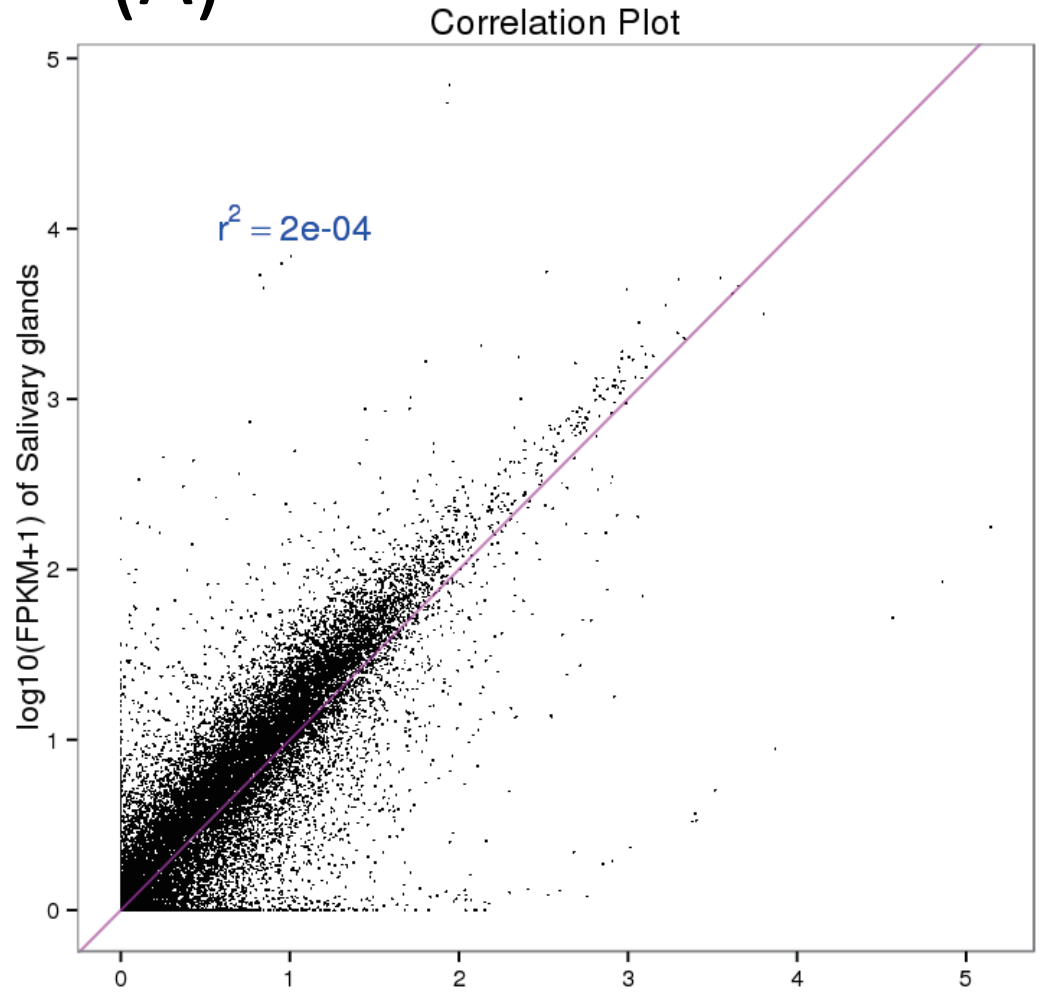

(D)

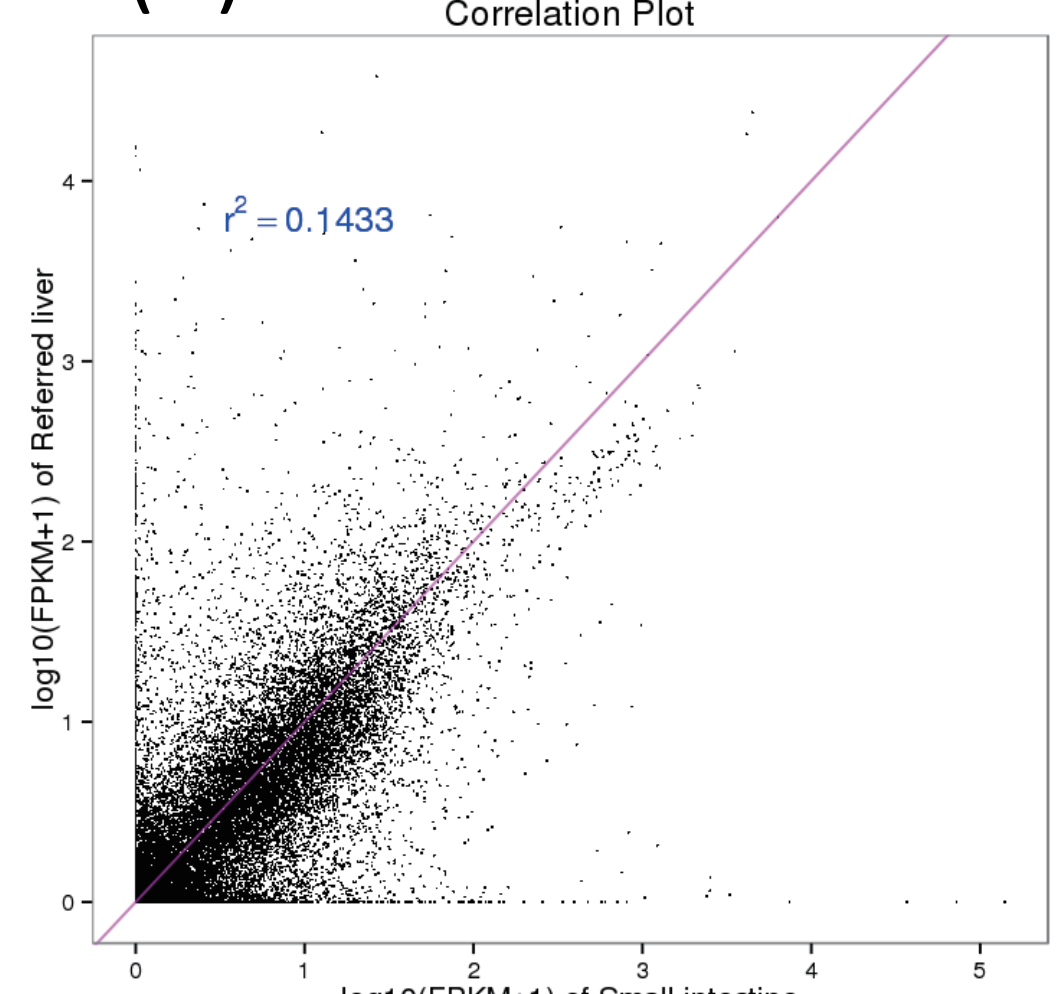

(B)

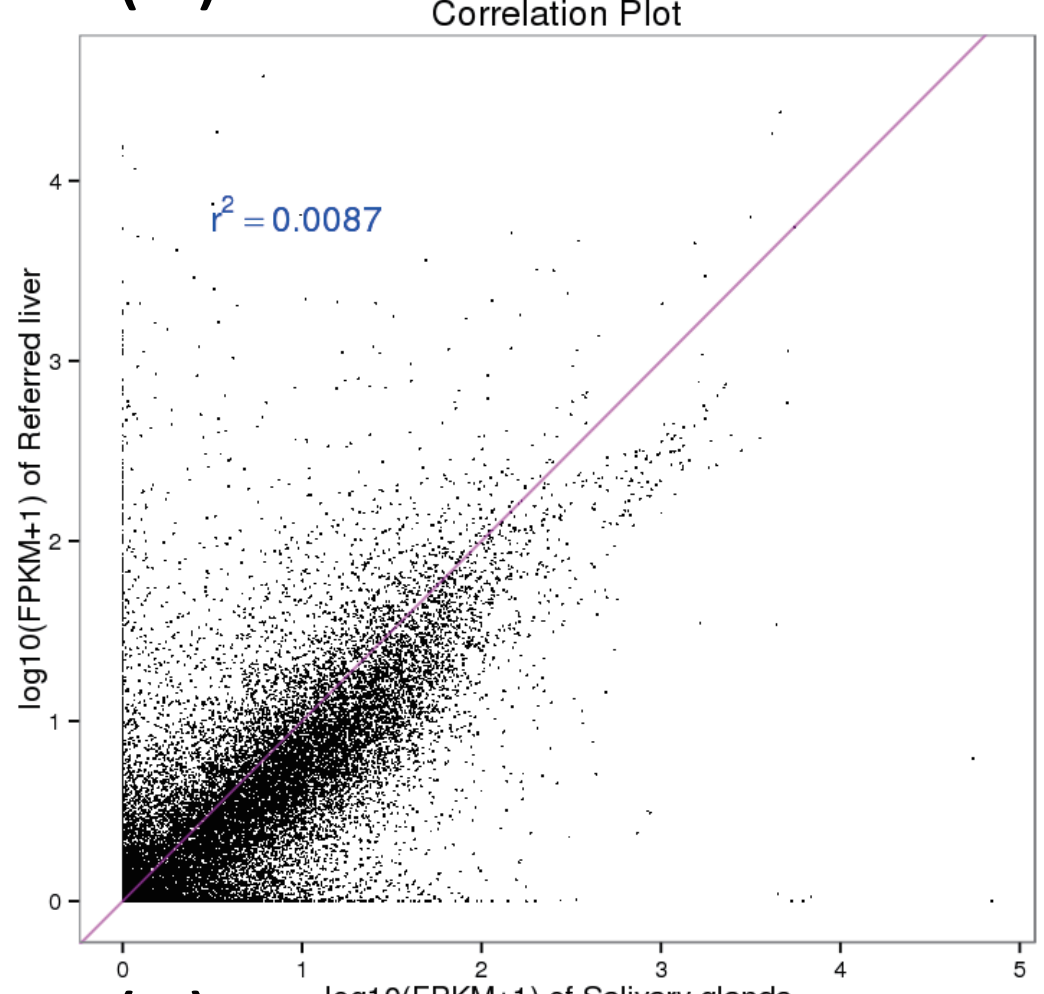

(E)

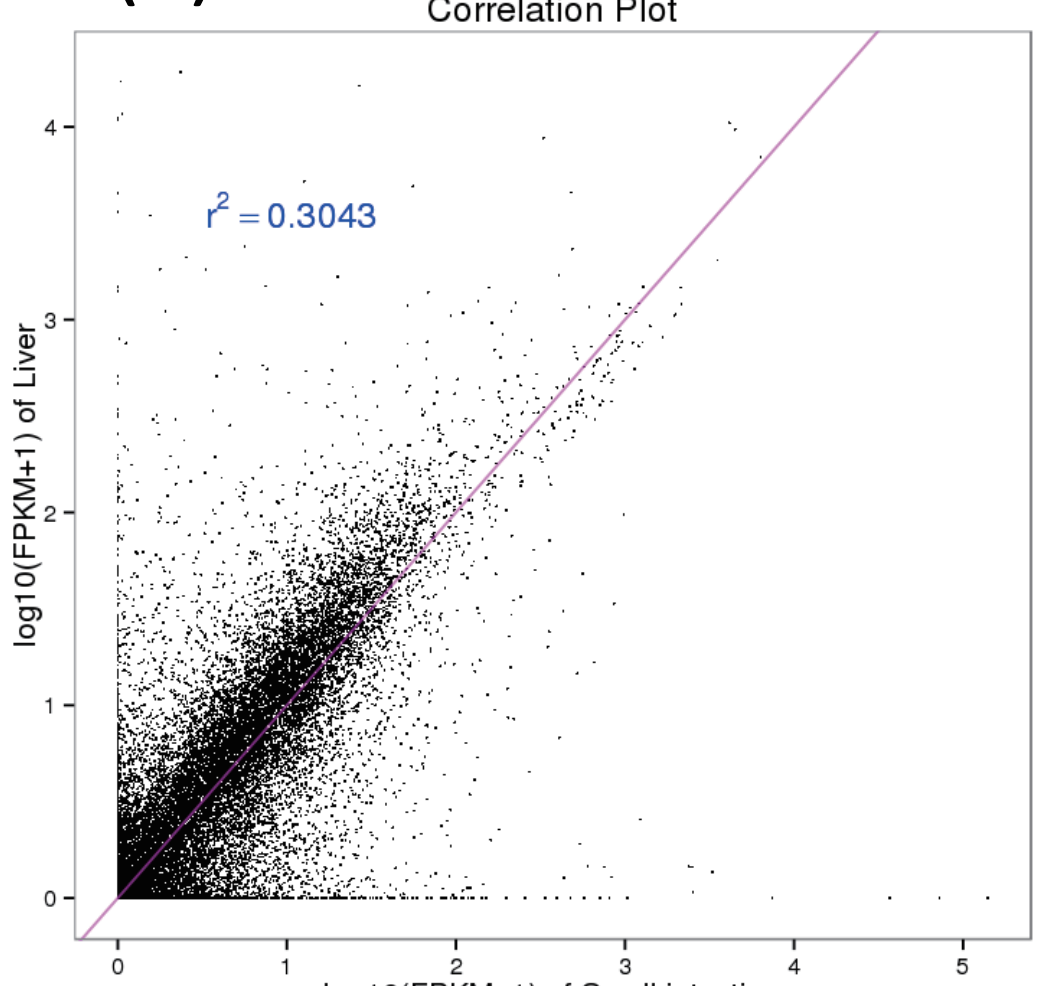

(C)

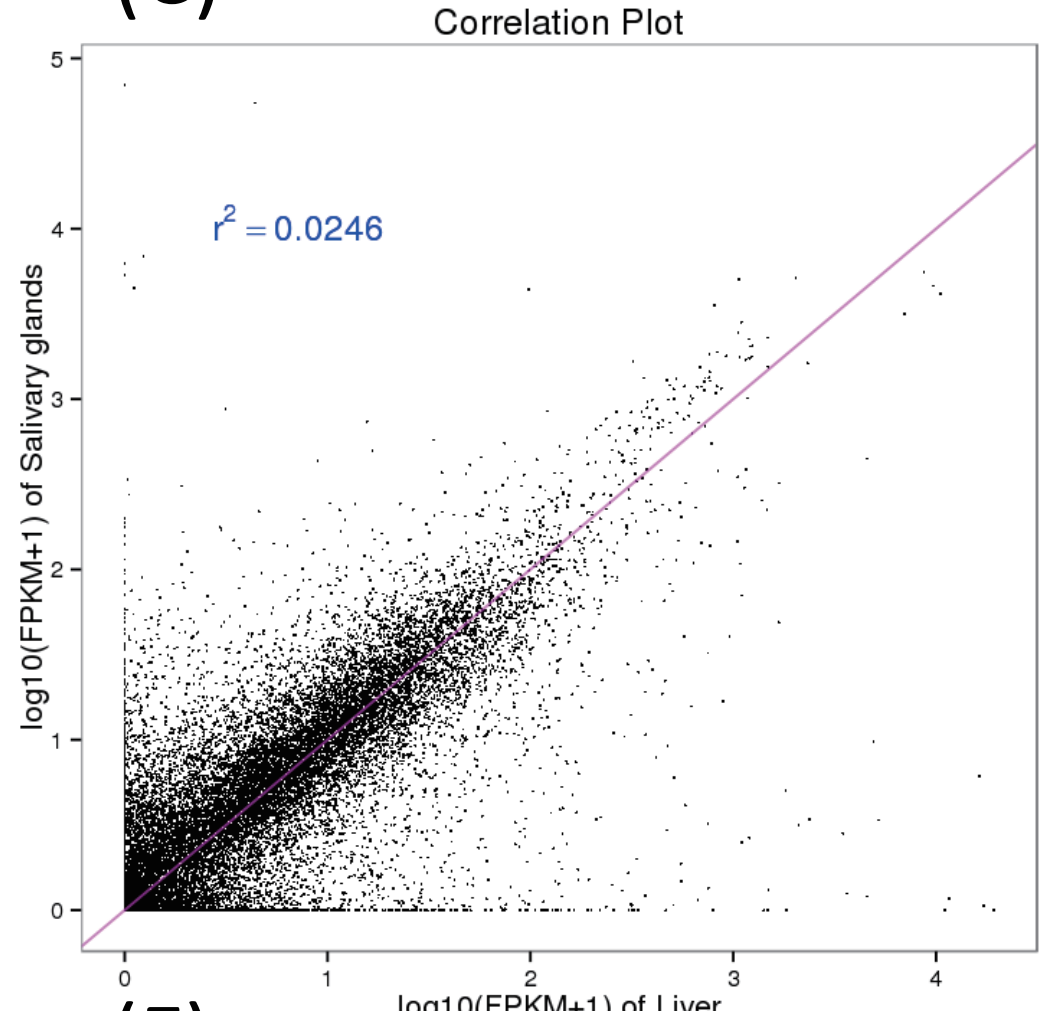

(F)

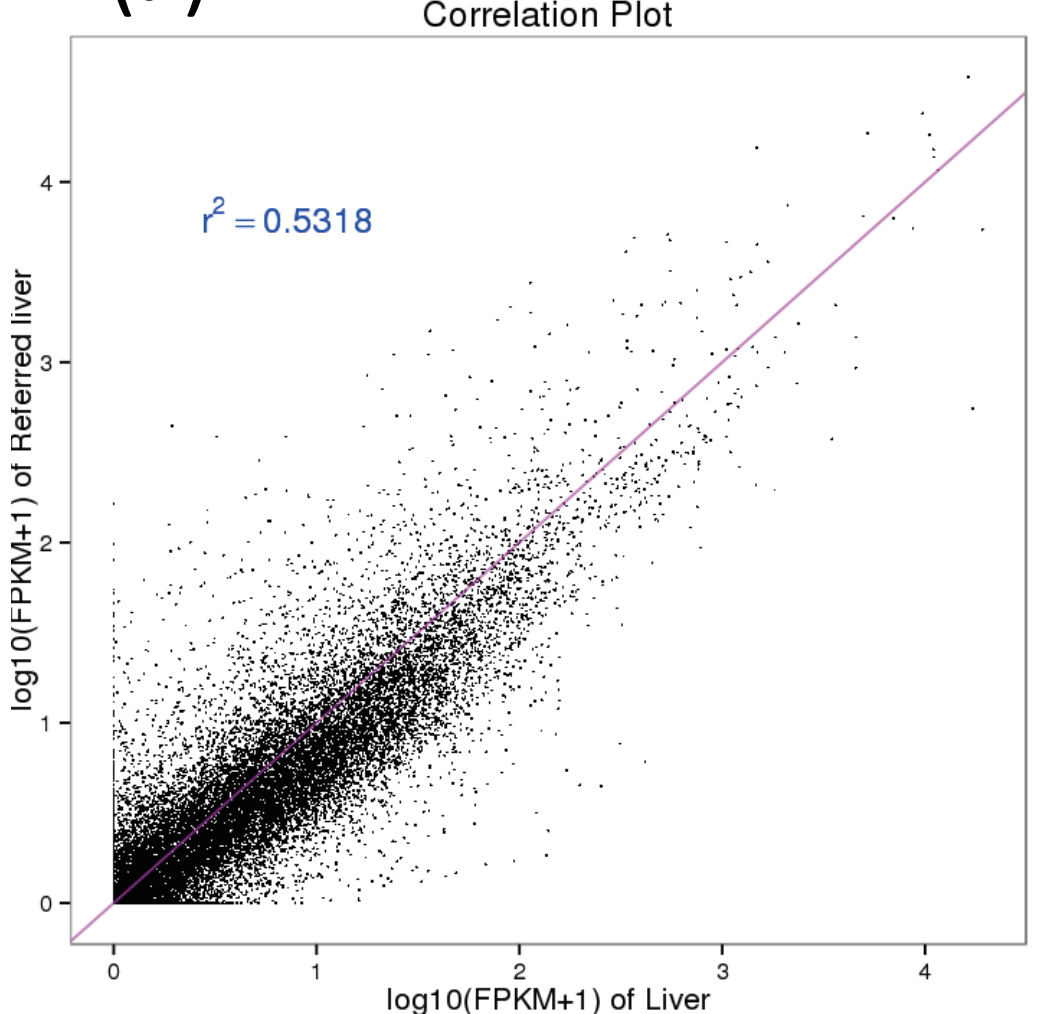




\section{Figure 4 (on next page)}

The transcripts related to metabolic pathways expressed in all three tissues.

The $x$-axis shows the number of transcripts with the KEGG function class, which was shown above the column, and the $y$-axis shows the KEGG function classes. 
PeerJ

Glyoxylate and dicarboxylate metabolism

Amino sugar and nucleotide sugar metabolism

Ascorbate and aldarate metabolism

Fructose and mannose metabolism

Pentose phosphate pathway

Glycolysis / Gluconeogenesis

alpha-Linolenic acid metabolism

Arachidonic acid metabolism

Ether lipid metabolism

Glycerolipid metabolism

Primary bile acid biosynthesis

Synthesis and degradation of ketone bodies

Fatty acid elongation

Phenylalanine, tyrosine and tryptophan biosynthesis

Phenylalanine metabolism

Histidine metabolism

Lysine degradation

Valine, leucine and isoleucine biosynthesis

Cysteine and methionine metabolism

Alanine, aspartate and glutamate metabolism

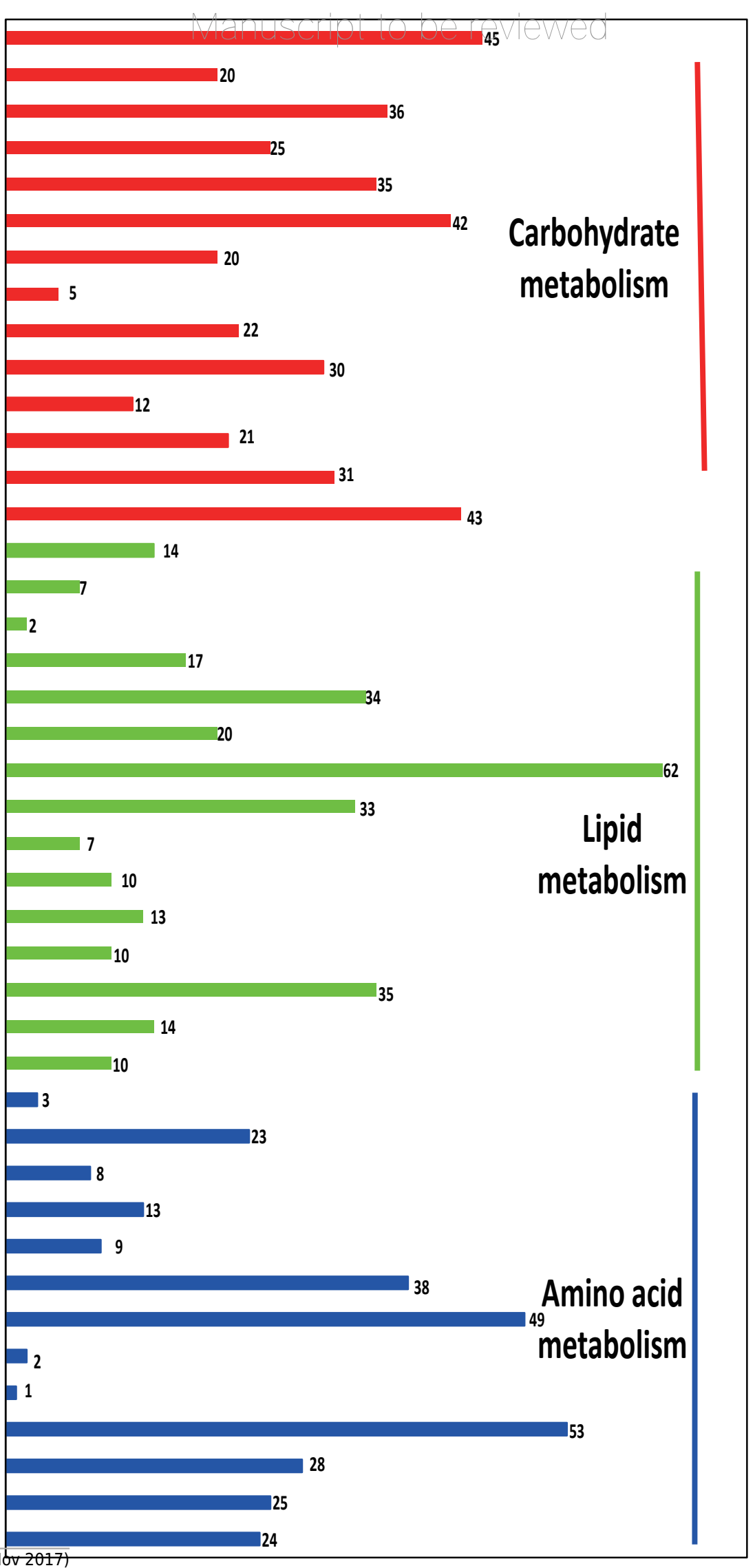


Figure $\mathbf{5}$ (on next page)

The numbers of transcripts related to metabolic pathways that are specifically expressed in the salivary glands, liver, and small intestine of adult female Malayan pangolin (Manis javanica).

The $x$-axis shows the KEGG function classes, and the $y$-axis shows the number of transcripts with the corresponding KEGG function class. 


\section{Table $\mathbf{1}$ (on next page)}

Sequencing statistics for the transcriptomes obtained from the salivary glands, liver, and small intestine of adult female Malayan pangolin (Manis javanica). 


\begin{tabular}{|c|c|c|c|c|c|}
\hline Organ & $\begin{array}{c}\text { Raw pairs } \\
\text { (bp) }\end{array}$ & $\begin{array}{c}\text { Clean pairs } \\
\text { (bp) }\end{array}$ & $\begin{array}{c}\text { Clean bases } \\
\text { (bp) }\end{array}$ & GC Content & $\% \geqslant Q 30 \%$ \\
\hline $\begin{array}{c}\text { Small } \\
\text { intestine }\end{array}$ & $20,296,915$ & $15,117,014$ & $4,535,104,200$ & $53.71 \%$ & $95.30 \%$ \\
\hline Liver & $33,288,751$ & $22,073,184$ & $6,621,955,200$ & $53.15 \%$ & $94.67 \%$ \\
\hline $\begin{array}{c}\text { Salivary } \\
\text { glands }\end{array}$ & $20,297,074$ & $14,857,037$ & $4,457,111,100$ & $51.33 \%$ & $95.58 \%$ \\
\hline $\begin{array}{c}\text { Referred } \\
\text { liver }\end{array}$ & - & $45,306,423$ & $9,061,284,600$ & $50.87 \%$ & $90.35 \%$ \\
\hline
\end{tabular}




\section{Table 2 (on next page)}

Summary of the sequencing data aligned to the Malayan pangolin (Manis javanica) whole genome reference sequence. 


\begin{tabular}{|c|c|c|c|c|}
\hline 2 & $\begin{array}{c}\text { Total } \\
\text { Reads }\end{array}$ & $\begin{array}{c}\text { Mapped } \\
\text { Reads }\end{array}$ & $\begin{array}{c}\text { Unique } \\
\text { Mapped Reads }\end{array}$ & $\begin{array}{c}\text { Multiple } \\
\text { Map Reads }\end{array}$ \\
\cline { 2 - 5 } Organ & $30,234,028$ & $22,277,733$ & $21,843,354$ & 434,379 \\
Small & $(73.68 \%)$ & $(72.25 \%)$ & $(1.44 \%)$ \\
\hline intestine & & $31,965,251$ & $31,694,559$ & 270,692 \\
Liver & $44,146,368$ & $(72.41 \%)$ & $(71.79 \%)$ & $(0.61 \%)$ \\
\hline Salivary & $29,714,074$ & $20,673,075$ & $19,911,660$ & 761,415 \\
glands & & $(69.57 \%)$ & $(67.01 \%)$ & $(2.56 \%)$ \\
\hline Reference & $90,612,846$ & $80,913,231$ & $80,064,920$ & 848,311 \\
liver & & $(89.30 \%)$ & $(88.36 \%)$ & $(0.94 \%)$ \\
\hline
\end{tabular}




\section{Table 3(on next page)}

Summary of genes related to the diet of the Malayan pangolin (Manis javanica). 


\begin{tabular}{|c|c|}
\hline Type & Gene name \\
\hline Opsin & GRK1, OPN1SW, OPN1LW, OPN4, PDE6D, PDE6G, PDE6H, RHO \\
\hline Taste & $\begin{array}{l}\text { TAS1R2,TAS1R3, TAS2R1, TAS2R4, TAS2R7, TAS2R10, TAS2R30, } \\
\text { TAS2R38, TAS2R40 }\end{array}$ \\
\hline Olfactory & $\begin{array}{l}\text { CNGA2, DTMT, OLF1, OLF2, OLF3, OLF4, OR1A1, OR1D2, OR1E1, } \\
\text { OR1E2, OR1E5, OR1G1, OR3A1, OR3A2, OR3A3 }\end{array}$ \\
\hline Carbohydrases & $\begin{array}{l}\text { AGL, AMY2, CHIA, CHI3L1, CHID1, GAA, GANAB, GANC, GBA3, } \\
\text { GLB1, GLB1L, PRKCSH, SI }\end{array}$ \\
\hline Lipases & $\begin{array}{l}\text { ABHD6, ABHD12, CEL, CLPS, DDHD1, GPLD1, Group XV } \\
\text { phospholipase A2, LIPA, LIPC, LIPE, LIPF, LIPH, LMF1, LMF2, LPL, } \\
\text { LYPLAL1, NAPEPLD, PLA1A,PLA2G1B, PLA2G2A, PLA2G3, } \\
\text { PLA2G4A, PLA2R1, PLB1,PLBD1, PLBD2, PLD3, PNLIP, PNLIPRP1, } \\
\text { PNLIPRP2, PNPLA2, PNPLA8 }\end{array}$ \\
\hline Protease & $\begin{array}{l}\text { Anionic trypsin, ANPEP, Cationic trypsin, CELA1, Chymotrypsin A } \\
\text { chain C, CTRB1, CTRC, DPP6, DNPEP, ENPEP, ERAP2, LAP3, } \\
\text { METAP1, METAP2, NPEPL1, PGC, PRSS12, Trypsin, XPNPEP1, } \\
\text { XPNPEP2, XPNPEP3 }\end{array}$ \\
\hline Transporters & $\begin{array}{l}\text { SLC1A1, SLC1A3, SLC1A6, SLC1A4, SLC1A5, SLC7A8, SLC43A2, } \\
\text { SLC6A15, SLC6A17, SLC6A19, SLC38A1, SLC38A2, SLC38A4, } \\
\text { SLC38A5, SLC38A7, SLC38A10, SLC38A11, SLC7A2, SLC7A14, } \\
\text { SLC7A11, SLC25A29, SLC2A1, SLC2A2, SLC2A3, SLC2A4, } \\
\text { SLC2A5, SLC2A8, SLC2A9, SLC2A12, SLC35A4, SLC35A5, } \\
\text { SLC50A1, SLC35A3, SLC35B4, SLC35D2, CLCN3, CLCN5, CLCN7, } \\
\text { MFSD5, MAGT1, MMGT1, MRS2, NIPA2, NIPAL1, Sodium- } \\
\text { independent sulfate anion transporter, SLC4A4, SLC20A1, SLC20A2, } \\
\text { SLCO1C1, SLCO3A1, SLCO4C1, LMBRD1, SLC5A6, SLC19A3, } \\
\text { SLC25A32, SLC52A2, SLC52A3, SLC5A1, SLC5A4, SLC5A10, }\end{array}$ \\
\hline
\end{tabular}




\begin{tabular}{|c|c|}
\hline & $\begin{array}{l}\text { SLC5A2, SLC28A1, SLC5A3, APOA1, APOA2, APOB, Apolipoprotein } \\
\text { A-IV, APOC2, APOC3, APOC4, APOD, APOE, APOM, APOO, } \\
\text { SLC6A2, SLC6A3, SLC6A4, SLC6A8, SLC6A9, SLC6A12, SLC6A13, } \\
\text { SLC10A2, SLC5A12, SLC16A1, SLC16A9, SLC16A13, SLC17A6, } \\
\text { SLC17A7, SLC26A2, SLC29A3, SLC44A2, SLC44A3, SLC44A4, } \\
\text { SLC44A5, SLC45A2, SLC46A2 }\end{array}$ \\
\hline
\end{tabular}

